\title{
Intrathoracal displacement of the humeral head after fracture
}

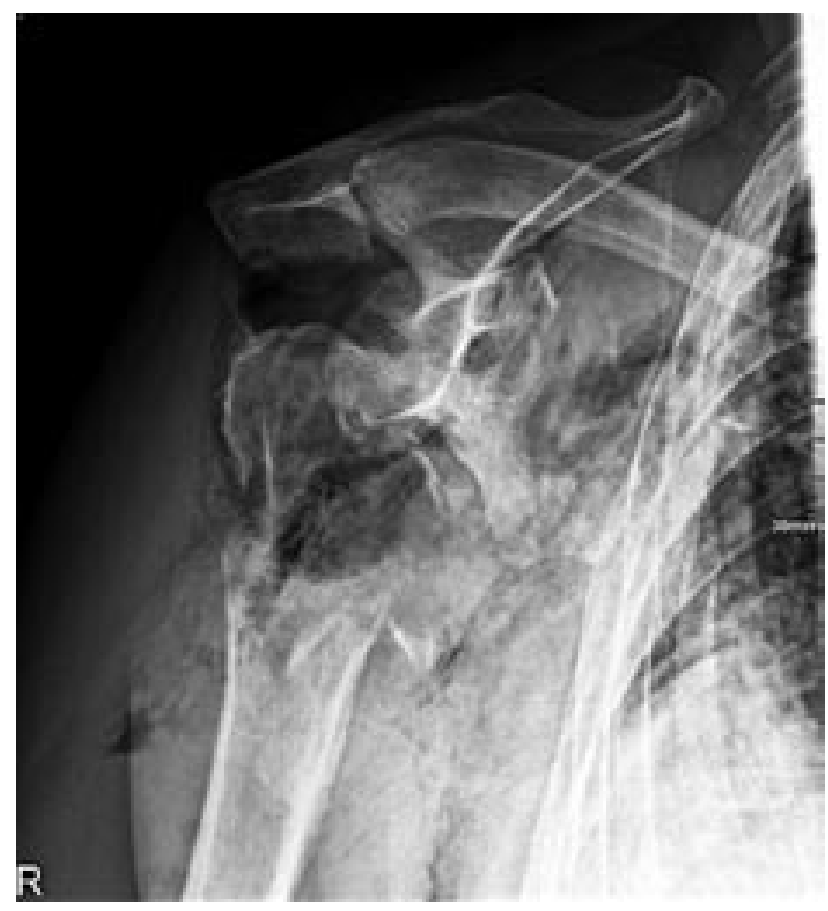

Fig. 1

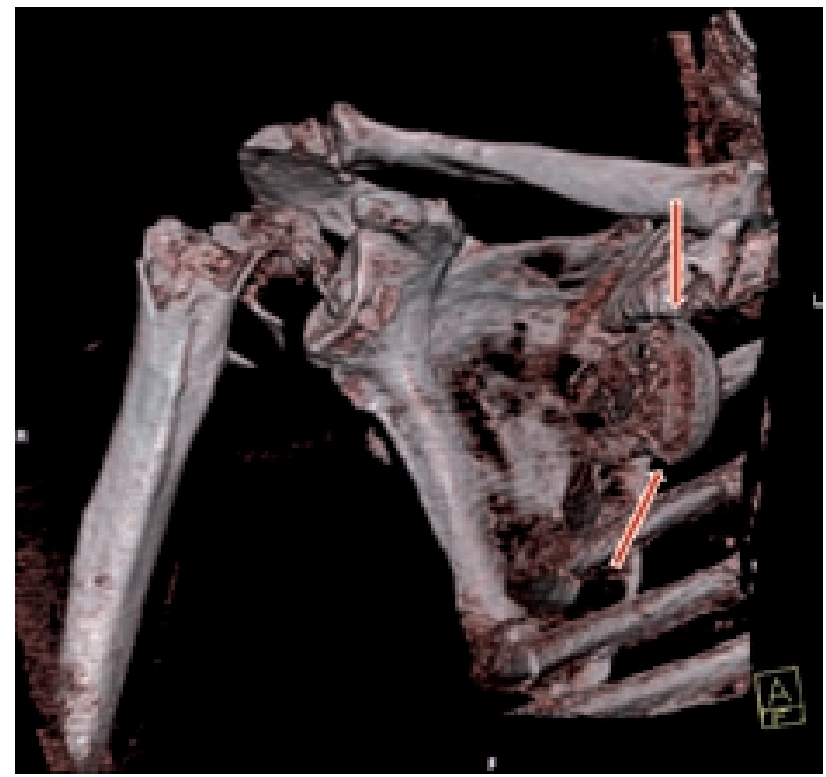

A 63-year-old male patient presented to the trauma surgery clinic because of serious right-sided shoulder pain combined with respiratory distress. He reported falling from a park bench while sleeping the night before. On plain X-ray examination a fractured right humerus with missing humeral head was noted (Fig. 1). Additionally, a serial rib fracture involving ribs $1-5$ on the right side was obvious. Thoracal x-ray showed a shadow in the right upper lobe of the lung. The subsequent 3-D reconstruction of CT scan revealed an intrathoracic traumatic displacement of the fractured humeral head into the right thoracic cavity (Fig. 2; arrows point to humeral head). Interestingly, no loss of sensibility on the affected right upper extremity was detectable, however, due to the injury hematothorax developed. In a first emergency procedure, the humeral head was removed through a right-sided thoracotomy facilitated through the serial rib fracture. After stabilization of the patient, prosthesis for shoulder joint reconstruction was performed successfully two weeks later. The further postoperative course was uneventful, and the patient was able to leave the hospital 14 days later.

This type of injury is exceedingly rare and only sparsely described in the existing literature [1-3], but should be suspected when high-impact trauma is present.

Harald Schoffl, Stefan M. Froschauer, and Georg M. Huemer

\section{References}

1. Harman BD, Miller NG, Probe RA (2004) Intrathoracic humeral head fracture-dislocation. J Orthop Trauma 18: $112-115$

2. Eberson CP, Ng T, Green A (2000) Contralateral intrathoracic displacement of the humeral head. A case report. J Bone Joint Surg Am 82: 105-108

3. Brogdon BG, Crotty JM, MacFeely L, McCann SB, Fitzgerald M (1995) Intrathoracic fracture-dislocation of the humerus. Skeletal Radiol 24: 383-385

Keywords: Fracture, humerus, thorax, displacement.

Correspondence: Georg M. Huemer, MD, Allgemeines Krankenhaus der Stadt Linz, Krankenhausstraße 9, 4020 Linz, Austria, E-mail: georg.huemer@maz.at

Fig. 2 